\title{
Observation of the enhanced backscattering of light by the end of a tilted dielectric cylinder owing to the caustic merging transition
}

\author{
Philip L. Marston, Yibing Zhang, and David B. Thiessen
}

\begin{abstract}
The scattering of light by obliquely illuminated circular dielectric cylinders was previously demonstrated to be enhanced by a merger of Airy caustics at a critical tilt angle. [Appl. Opt. 37, 1534 (1998)]. A related enhancement is demonstrated here for backward and near-backward scattering for cylinders cut with a flat end perpendicular to the cylinder's axis. It is expected that merged caustics will enhance the backscattering by clouds of randomly oriented circular cylinders that have appropriately flat ends. (C) 2003 Optical Society of America

OCIS codes: $\quad 290.5850,010.2940,290.1350,080.1510,060.2300$.
\end{abstract}

\section{Introduction}

When light is incident upon a tilted transparent circular cylinder, the scattering angle of the Airy caustic that corresponds to the primary rainbow depends on the tilt angle of the cylinder. ${ }^{1-4}$ The evolution of the associated Airy pattern in far-field scattering was previously observed by recording of the scattering of laser light by a long cylindrical fiber over a range of tilt angles. ${ }^{4}$ Whereas this phenomenon is of general interest in scattering research, ${ }^{5-8}$ it may be relevant to the scattering by naturally produced circular cylinders of ice viewed with the unaided eye. ${ }^{3}$ Here we describe how backscattering can be enhanced when the cylinder is bluntly truncated and tilted at (or close to) the critical tilt angle associated with the merging of the Airy caustics. Although these observations were made with a fused-silica fiber, it is widely realized that internal reflections off the blunt truncations of ice crystals contribute to scattering enhancements that are visible to the unaided eye. ${ }^{9-11}$ Most observations of naturally produced ice crystals are not of

When this research was performed, the authors were with the Department of Physics, Washington State University, Pullman, Washington 99164-2814. Y. Zhang is now with the Center for Photonics Technology, Bradley Department of Electrical and Computer Engineering, Virginia Polytechnic Institute and State University, Blacksburg, Virginia 24061. P. L. Marston's e-mail address is marston@wsu.edu.

Received 14 January 2002; revised manuscript received 29 March 2002.

0003-6935/03/030412-06\$15.00/0

(C) 2003 Optical Society of America backscattering, but there is evidence that ice in cirrus clouds can produce enhanced backscattering that is visible to the unaided eye. ${ }^{12}$

The geometry of the scattering processes considered is shown in Fig. 1, where the notation is like that used in Ref. 3. Unlike the situation considered in Ref. 3, however, here the internal reflection from the blunt lower end of the cylinder is considered. The associated ray theory was developed by Blonigen and Marston $^{8}$ (the specific application was to the backscattering of sound by plastic cylinders in water). Only the results that are essential to explaining the current experiments are described here. The important result is that, when the truncation of the cylinder is flat and at $90^{\circ}$ with respect to the cylinder's axis, the projection of the rays on the base of the cylinder is the same as for the rays considered in Ref. 3 , for which rays do not reflect from a truncation. This is evident when rays reflected from the truncation are replaced with rays in an image cylinder. The latter rays generate conical scattered wave fronts, as explained in Refs. 3 and 4 for the infinite cylinder problem.

Figure 2 shows the special case of the rays that lie in the meridional plane defined by the incident wave vector and the cylinder's axis. As a consequence of the reversibility of the rays, there is a contribution to the backscattering that may be present at all tilt angles. Ordinarily, however, this contribution is expected to be weak because of the curvature of the outgoing wave front orthogonal to the plane of the figure. In the special case of a cylinder tilted at the angle where the Airy caustics associated with the 


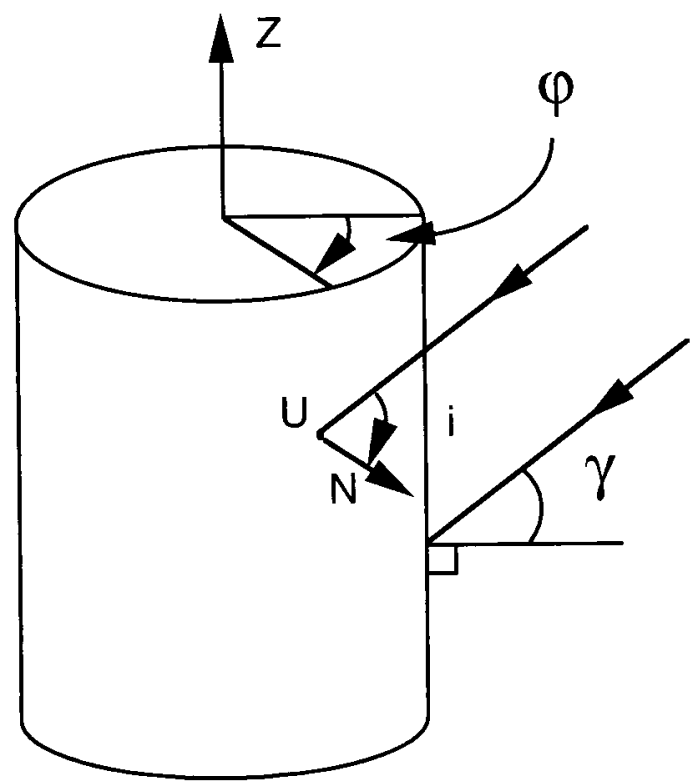

Fig. 1. Geometry of the truncated circular cylinder considered. The ends of the cylinder are perpendicular to the symmetry axis. The wave vector of the illumination is tilted by an angle $\gamma$ relative to the ends of the cylinder. The actual angle of incidence is $i$ for an illuminated surface point $U$ (where the local cylinder normal is $N)$. The angle of incidence projected onto the base plane is $\varphi$, which is smaller than $i$

cylinder's primary rainbow merge in the meridional plane, the aforementioned wave-front curvature vanishes. (See Fig. 7 of Ref. 8 or App. A of Ref. 3). Consequently, the associated backscattering is expected to be significantly enhanced. Whereas the curvature of the backscattered wave front always vanishes in the plane of Fig. 2, it vanishes only for the orthogonal plane at the critical tilt angle. When this condition holds, Blonigen and Marston ${ }^{8}$ show that the far-field scattering amplitude (the electric field amplitude in the present context of light scattering) is proportional to $(k a)^{3 / 4} a$, where $a$ is the radius of the cylinder and $k=2 \pi / \lambda$ is the wavelength of the illumination. It was demonstrated in Ref. 8 that $k a$ need not be extremely large for this enhancement to be significant in the backscattering of sound. The acoustic signature of this enhancement was easily observed for $k a$ as small as 8 (which corresponds to the lowest value of $k a$ that could easily be investigated). When the tilt angle is decreased below the critical value, the backscattering enhancement does not disappear abruptly because the observer is in an illuminated region of the Airy caustic. The critical tilt angle is given by ${ }^{3,4}$

$$
\gamma_{c}=\arccos \left\{\left[\left(n^{2}-1\right) / 3\right]^{1 / 2}\right\},
$$

where $n$ is the refractive index of the cylinder relative to that of the surrounding air. We derive this condition by requiring that the Bravais effective refractive index,

$$
n^{\prime}(\gamma, n)=\left(n^{2}-\sin ^{2} \gamma\right)^{1 / 2} / \cos \gamma \geq n,
$$

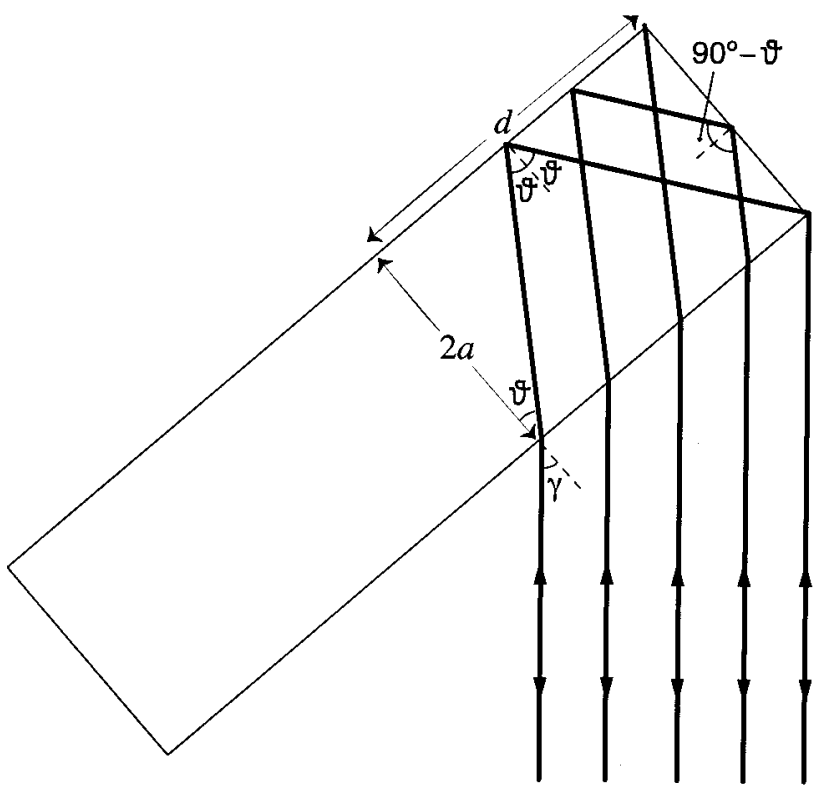

Fig. 2. Rays confined to the meridional plane of the cylinder that contribute to the backscattering after the fewest number of reflections. The rays shown are reversible. From Ref. 8, only the region within a distance $d=4 a(\tan \gamma) / n^{\prime}$ contributes to the backscattering for the process considered, where $n^{\prime}$ is given by Eq. (2). Rays can be backscattered after additional internal reflections; however, the contributions from those rays are relatively weak near tilts given by Eq. (1). This is a consequence of the curvature of the outgoing wave fronts associated with the extra reflections. Angle of refraction $\vartheta$ is discussed in Section 5 below.

take on the value 2 such that the Airy caustics merge.

The projections of the rays onto the base plane are evaluated by considering rays normally incident on a cylinder having a refractive index $n^{\prime}$. This follows in the description of rays from the invariance of the axial projection of the wave number in the case of a tilted cylinder, as shown in App. A of Ref. 8. The projection of the scattering angle onto the base plane is related to the projected angle of incidence $(\varphi$ in Fig. 1 ) by Snell's law and by the usual geometric condition [Eq. (2) of Ref. 3]. The Descartes ray is given by the usual condition:

$$
\cos ^{2} \varphi_{D}=\left(n^{\prime 2}-1\right) / 3,
$$

and $\varphi_{D}$ vanishes as $n^{\prime}$ approaches 2 . Consequently, when $\gamma=\gamma_{c}$ the rays in Fig. 2 are the Descartes rays that have merged in the meridional plane. For a circular cylinder of ice when birefringence is neglected, $n=1.31$, which is the value for yellow light. The experiments described here were made with red light and a fused-silica cylinder, so $n=1.447$. The corresponding critical tilt angles from Eq. (1) are $\gamma_{c}$ of $60.8^{\circ}$ and $52.3^{\circ}$, respectively. When the cylinder is not tilted, the Descartes scattering angles $\theta_{D}$ are $134.5^{\circ}$ and $153.0^{\circ}$.

\section{Apparatus and Observations}

The phenomena considered concern the internal reflection from only one end of the circular cylinder, so 


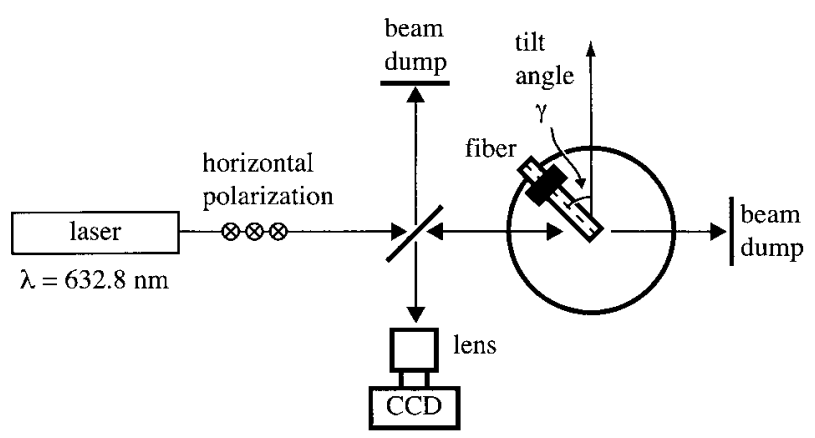

Fig. 3. Experimental configuration for viewing the backscattering from the end of a small tilted cylinder (not drawn to scale). A lens is placed in front of the CCD camera with the CCD array at the focal plane. The backscattering region was also viewed by eye through a telescope. The illumination is horizontal, and the axis of the cylinder is tilted from the vertical by an amount $\gamma$ by a goniometer. The goniometer measures the rotation about a horizontal axis that is perpendicular to the incident wave vector.

it was necessary only to prepare a cylinder that had one flat end. The cylinder was a homogenous (unclad) fused-silica fiber manufactured for other purposes. One end of the fiber was cut flat and polished. The diameter was $2 a=1.000 \mathrm{~mm}$. Although inspection with a microscope indicated that the truncation appeared to be perpendicular to the axis of the fiber, equipment to resolve any error in angle of this truncation or to measure any residual curvature in the end was not available. The fiber was mounted in a stage such that tilt angle $\gamma$ could be adjusted as shown in Fig. 3.

The end region of the fiber was illuminated by a beam from a polarized $\mathrm{He}-\mathrm{Ne}$ laser with a wavelength $\lambda=632.8 \mathrm{~mm}$. The $E$-field vector of the illumination was perpendicular to the plane defined by the incident wave vector and the fiber's axis. Nearbackward scattering was observed through a beam splitter. The experimental arrangement is similar to one used for observing glory scattering by bubbles in liquids. ${ }^{13}$ We took care to avoid spurious reflections by placing black felt in the path of the extra reflection from the beam splitter as well as in the region of the near-forward scattering. The far-field backscattering pattern was observed in three ways: (i) through a telescope focused on infinity, (ii) with a CCD camera at the focal plane of a lens that had a focal length of $85 \mathrm{~mm}$, and (iii) with a camera with the CCD in instance (ii) replaced with photographic film. Each of the methods of observation revealed a significant near-backward scattering enhancement when tilt angle $\gamma$ was close to the predicted $\gamma_{c}$ of $52.3^{\circ}$. To determine the actual direction of true backscattering we temporarily placed a diffuse reflector in the path of the extra beam-splitter reflection (shown as the upward-pointing arrow in Fig. 3) at a large distance away. It was also possible to optically determine the orientation of the cylinder that corresponded to $\gamma=$ $0^{\circ}$. We verified the angle calibration of the CCD image (from the focal length of the lens) within $1 \%$ by viewing a distant object of known angular width.

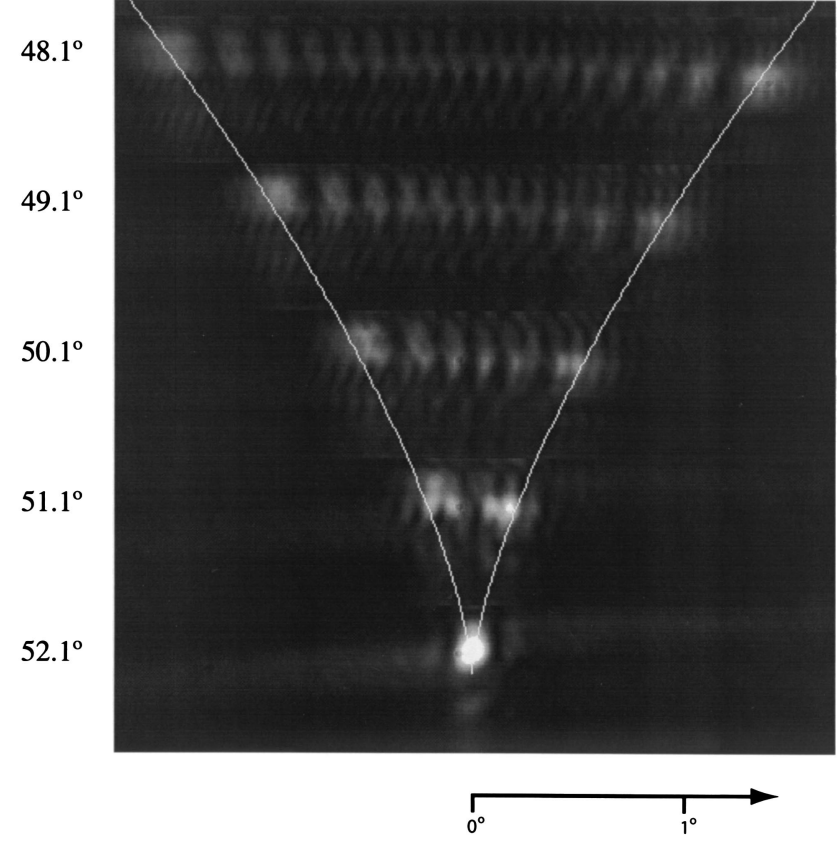

Fig. 4. CCD strip records of the near-backward scattering for the value of $\gamma$ displayed at the left. The strips are shifted vertically in proportion to tilt deviation $\gamma_{c}-\gamma$. The evolution as a function of tilt for the calculated Descartes condition is shown as white curves on the right and left sides of the pattern. This evolution was calculated as explained in Section 3. The horizontal scale shows a $1^{\circ}$ deviation in the backscattering angle. The CCD camera has been saturated near the brightest region of two of the records.

The scattering patterns observed were similar for the three methods of observation described above, and it is convenient to display here only representative results recorded by the CCD camera. The relevant scattering is in a narrow angular strip because, as shown in Fig. 2, there is insignificant spreading of the rays in the meridional plane. We display the evolution of the scattering pattern with tilt by offsetting the strips by an amount proportional to the shift in $\gamma$, as shown in Fig. 4. It is evident from inspection of Fig. 4 that, as $\gamma$ is reduced below a critical value, bright regions that correspond to the Airy caustics from each side of the cylinder shift away from the meridional plane. It must be emphasized, however, that the actual pattern does not have the vertical shift shown in Fig. 4. (This is in contrast to Fig. 4 of Ref. 4, where the vertical location of the scattering pattern shifted with tilt angle for the reasons explained there). The brightest scattering is observed when $\gamma=52.1^{\circ}$, which is interpreted as the measured critical tilt angle. Observations (not shown in Fig. 4) confirm that the scattering near the meridional plane decreases rapidly after $\gamma$ is raised above the predicted $\gamma_{c}$ of $52.3^{\circ}$.

The enhanced scattering near the caustic saturated the CCD camera in Fig. 4. The camera's sensitivity was sufficient to permit the camera to record the scattering pattern away from the caustic. Inspection of Fig. 4 shows that the saturation is most pronounced close to the critical tilt angle; however, 
the presence of saturation does not call into question the general properties of the observed scattering.

\section{Evolution of the Airy Caustic as the Cylinder Is Tilted}

Figure 4 also displays a prediction of the offset of the Airy caustic from backscattering as a function of the tilt of the cylinder. The procedure for calculating this cusp-shaped curve is summarized as follows: First, scattering angle $\theta_{D}$ for the Descartes ray projected onto the base plane of the cylinder is determined as a function of tilt angle as explained in Sec. 2 of Ref. 3. The deviation angle from backscattering from the perspective of the observer (the narrow strip of the scattering recorded by the camera) differs from the base-projected direction, as we explain in Appendix A. From the transformation derived in Appen$\operatorname{dix} \mathrm{A}$, the evolution of the backscattering angle for the Descartes ray is calculated, and this is the prediction plotted in Fig. 4 as a function of a continuous tilt angle $\gamma$. The strips displayed correspond to discrete samples of $\gamma$ along this curve near the midportion of each strip. It is a well-known property of caustics that the brightest portion of the scattering pattern is shifted to the interior of the caustic. ${ }^{14}$ This is confirmed to be the case in Fig. 4 for the backscattering pattern of the truncated cylinder.

\section{Supernumerary Oscillations}

Except when $\gamma$ lies close to $\gamma_{c}$, inspection of Fig. 4 suggests the presence of features analogous to supernumerary rainbows. These features are expected to correspond to Airy function extrema that are offset from the most prominent maximum. (In Fig. 4 these features are not to be confused with a finer structure that is also visible. The properties of the fine structure changed for slightly different experimental setups, and those features were not investigated). The correspondence of the broad features to the supernumerary features of the Airy function ${ }^{15}$ was confirmed by an approximate analysis. The analysis concerns the simplest observable, which is the difference in the backscattering angles that corresponds to the adjacent nulls in the Airy pattern closest to the caustic. For $\gamma$ of $48.1^{\circ}$, these angles are measured to be $0.23^{\circ}$.

This angle shift may be calculated as follows: First, the corresponding spread in the scattering angles projected onto the base of the cylinder is determined by use of the physical optics model of the rainbow. ${ }^{15,16}$ The scattering amplitude near an Airy caustic varies in proportion to $\mathrm{Ai}(w)$, where $\mathrm{Ai}$ is the Airy function in contemporary notation ${ }^{14,16}$ and the argument $w$ is proportional to the deviation from the Descartes angle. In the present application to a tilted cylinder, $w$ is proportional to $\theta-\theta_{D}$, where $\theta$ and $\theta_{D}$ correspond to scattering angles and Descartes angles, respectively, that are projected onto the base of the cylinder. As functions of $\gamma, \theta_{D}$ and the projected angle of incidence are calculated as explained in Sec. 2 of Ref. 3. For a spherical drop or an untilted circular cylinder, $w$ may be compactly expressed by use of ${ }^{16} \sin \left(\varphi_{D}\right)$ and $\cos \left(\varphi_{D}\right)$. Application of the ray tracing procedure for tilted cylinders based on the effective index $n^{\prime}$ describes the intersection of the outgoing wave front with planes parallel to the base of the cylinder. From the dependence of $w$ on wave-front properties, the compact result from Sec. 10.4 of Ref. 16 gives

$$
w=\left(2 k_{r} a\right)^{2 / 3}\left(3 \sin \varphi_{D}\right)^{-1 / 3}\left(\cos \varphi_{D}\right)\left(\theta-\theta_{D}\right),
$$

where $\varphi_{D}$ and $\theta_{D}$ depend on $\gamma$ and $k_{r}$ is the reduced wave number, $k_{r}=k \cos \gamma$. (The use of the reduced wave number is an approximation. In the analogy of an infinite cylinder, the axial component of the wave number is a true invariant; however, here the axial component is affected by diffraction). The first two values of $w$ for which $\operatorname{Ai}(w)=0$ are -2.338 and -4.088 , which gives an increment in $w$ of 1.75 . For the conditions of this experiment, the corresponding increment in $\theta$ is $0.330^{\circ}$ for $\gamma$ of $48.1^{\circ}$. Finally, it is necessary to transform this increment as explained in Appendix A, where to simplify the analysis it may be assumed that the backscattering angle is small. The resultant predicted increment between the adjacent nulls is $0.221^{\circ}$. The measured increment is within $4 \%$ of the predicted increment. If the reduced wave number $k_{r}$ in Eq. (4) is replaced by $k$, the measured increment is approximately $136 \%$ of the modified prediction.

There is strong support for identifying the broad features as associated with supernumerary oscillations. The model summarized also predicts that the spacing will decrease with increasing $\gamma$, in agreement with the trend in Fig. 4. The discussion leading to an amplitude proportional to $\mathrm{Ai}(w)$ is applicable only close to the Airy caustic because (along with other approximations) contributions associated with any rays from the opposite half of the cylinder are neglected. It is shown in Ref. 8 that the scattering into the meridional plane in the analogous acoustical situation may be expressed by use of a Pearcey integral. It is plausible that, when $\gamma$ is close to $\gamma_{c}$, the lightscattering pattern can be approximated by use of a Pearcey integral. See also App. A of Ref. 3.

\section{Conclusions and Discussion}

These observations of the evolution of the pattern of the light scattered in the near-backward direction reveal a prominent enhancement in scattering when the tilt angle is close to a critical value predicted from geometrical considerations. Figure 4 shows that the pattern evolves in the generic way expected for the merging of Airy caustics and that the observed merger occurs with a tilt within $1^{\circ}$ of the predicted value. The observations reported here were made for horizontal polarization of the illumination, as indicated in Fig. 3. It may be anticipated that the magnitude and details of the pattern will depend on the polarization. Though the wave-number-radius product $k a$ was large in these experiments ( $k a=$ 4965), for the previously reported enhancements in acoustics experiments $k a$ was as small as 8. This result suggests that the phenomenon may be present in the backscattering by bluntly truncated dielectric 
cylinders over a wide range of $k a$. When $\gamma=\gamma_{c}$, the backscattered irradiance is expected to scale in proportion to $a^{2}(k a)^{3 / 2}$ when three-dimensional spreading is taken into account. ${ }^{8}$ By comparison, for the two-dimensional situation discussed in App. A of Ref. 3 the irradiance was expected to scale as $a(k a)^{1 / 2}$. (The additional factor of $k a^{2}$ occurs in the threedimensional case because the diffraction that contributes to the width of the strips in Fig. 4 is less pronounced when $k$ is large). Away from the condition $\gamma=\gamma_{c}$, the angular spacing of the supernumerary features that are visible in Fig. 4 are predicted from Eq. (4) to decrease in proportion to $(k a)^{-2 / 3}$ as $k a$ increases. The analogy between acoustical and optical scattering patterns ${ }^{14}$ indicates that the acoustical scattering pattern for the situation considered in Ref. 8 should depend on $\left(\gamma_{c}-\gamma\right)$ in a way generally similar to that of Fig. 4 with the diffraction features scaled for the appropriate $k a$.

The scattering enhancement described here is expected to contribute to the backscattering of light by clouds of bluntly truncated circular cylinders of ice. If the cylinders are randomly oriented, for visible light the cylinders tilted near $60.8^{\circ}$ are predicted to give the strongest backscattering by this mechanism. Whereas it may be uncertain whether such clouds occur naturally, some relevant scattering properties have been addressed by several studies concerned with atmospheric optics. ${ }^{17-21}$ The results of the present investigation, however, appear to be new.

The internal reflection from the end of the cylinder will be partial unless the internal angle of incidence exceeds $\arcsin (1 / n)$. In Fig. 2 this angle is denoted $90^{\circ}-\vartheta$, where $\vartheta$ is related to $\gamma$ by Snell's law: $\sin \gamma=$ $n \sin \vartheta$. When tilt $\gamma$ is the critical value given by Eq. (1), the reflection is total if $n$ exceeds 1.517. Consequently, there is only partial internal reflection for ice and for the fused-silica fiber that we viewed. Even if the reflection is only partial at $\gamma_{c}$, it is possible to have total internal reflection for the smaller values of $\gamma$ within the region of the cusp plotted in Fig. 4.

\section{Appendix A: Transformation of the Scattering Angle}

To compare the observations of the near-backward scattering with predictions, we need to give some consideration to the geometry. A cone of rays leaves the cylinder with the apex half-angle of the cone given by $\gamma^{\prime}=90^{\circ}-\gamma$, where $\gamma$ is the tilt shown in Fig. 1. As explained in Sections 1 and 4, features of the scattering may be predicted as functions of the direction of the projection onto the base plane. Let $\theta$ denote this projected angle, where $\theta=0$ give forward scattering into the meridional plane. It is convenient to introduce spherical coordinates in which $\theta^{\prime}=$ $180^{\circ}-\theta$ denotes an azimuthal angle and $\gamma^{\prime}$ is the polar angle relative to the cylinder's axis. We introduce Cartesian coordinates, where $x$ and $y$ are in the base plane of the cylinder and $z$ is along the cylinder's axis. The Cartesian unit vector for the direction $\left(\theta^{\prime}\right.$, $\left.\gamma^{\prime}\right)$ is $(x, y, z)=\left(\sin \theta^{\prime} \sin \gamma^{\prime}, \cos \theta^{\prime} \sin \gamma^{\prime}, \cos \gamma^{\prime}\right)$. Denote this direction by the unit vector $\hat{m}$. The unit vector for backscattering is in the direction $\hat{m}_{b}$, which has $\theta^{\prime}=0$ and $(x, y, z)=\left(0, \sin \gamma^{\prime}, \cos \gamma^{\prime}\right)$. The backscattering angle is given by

$$
\begin{aligned}
\psi\left(\theta^{\prime}, \gamma^{\prime}\right) & =\arccos \left(\hat{m} \cdot \hat{m}_{b}\right) \\
& =\arccos \left(\cos \theta^{\prime} \sin ^{2} \gamma^{\prime}+\cos ^{2} \gamma^{\prime}\right) .
\end{aligned}
$$

For $\gamma=0, \psi$ becomes $\theta^{\prime}$, as expected. From Eq. (A1) another example that follows from elementary considerations is that $\psi$ approaches $2 \gamma^{\prime}$ when $\theta^{\prime}$ approaches $180^{\circ}$. We arrived at the curve in Fig. 4 by plotting $\psi\left(180^{\circ}-\theta_{D}, 90^{\circ}-\gamma\right)$ on the horizontal axis as a continuous function of $\gamma$ along the vertical axis. It may be shown that this method of comparing measurements neglects the slightly arched appearance of the patterns. The correction associated with that curvature is negligible for the range of $\gamma$ considered.

This research was partially supported by the Office of Naval Research.

\section{References}

1. J. A. Lock and C. L. Adler, "Debye series analysis of the first order rainbow produced in scattering of a diagonally incident plane wave by a circular cylinder," J. Opt. Soc. Am. A 14, 1316-1328 (1997).

2. C. L. Adler, J. A. Lock, B. R. Stone, and C. J. Garcia, "Higherorder interior caustics produced in scattering of diagonally incident plane wave by a circular cylinder," J. Opt. Soc. Am. A 14, 1305-1315 (1997).

3. P. L. Marston, "Descartes glare points in scattering by icicles: color photographs and a tilted dielectric cylinder model of caustic and glare point evolution," Appl. Opt. 37, 1551-1556 (1998).

4. C. M. Mount, D. B. Thiessen, and P. L. Marston, "Scattering observations for tilted transparent fibers: evolution of Airy caustics with cylinder tilt and the caustic merging transition," Appl. Opt. 37, 1534-1539 (1998).

5. L. Mees, K. F. Ren, G. Grehan, and G. Gouesbet, "Scattering of a Gaussian beam by an infinite cylinder with arbitrary location and arbitrary orientation: numerical results," Appl. Opt. 38, 1867-1876 (1999).

6. J. A. Lock, C. L. Adler, and E. A. Hovenac, "Exterior caustics produced in scattering of a diagonally incident plane wave by a circular cylinder: semiclassical scattering theory analysis," J. Opt. Soc. Am. 17, 1846-1856 (2000).

7. P. L. Marston, "Optical caustics and related phenomena," in On Minnaert's Shoulders: Twenty Years of Light and Color Conferences, C. L. Adler, ed., Vol. 1 of Classic Reprints on CD-ROM (Optical Society of America, Washington, D.C., 2000).

8. F. J. Blonigen and P. L. Marston, "Backscattering enhancements for tilted solid plastic cylinders in water due to the caustic merging transition: observations and theory," J. Acoust. Soc. Am. 107, 689-698 (2000).

9. D. K. Lynch and W. Livingston, Color and Light in Nature (Cambridge U. Press, Cambridge, 1995).

10. W. Tape, Atmospheric Halos, Vol. 64 of Antarctic Research Series (American Geophysical Union, Washington, D.C., 1994).

11. K. N. Liou, Y. Takano, and P. Yang, "Light scattering and radiative transfer in ice crystal clouds: Applications to climate research," in Light Scattering by Nonspherical Particles, M. I. Mishchenko, J. W. Hovenier, and L. D. Travis, eds. (Academic, San Diego, Calif., 2000), Chap. 15, pp. 417-449.

12. K. Sassen, W. P. Arnott, J. M. Barnett, and S. Aulenbach, "Can cirrus clouds produce glories?” Appl. Opt. 37, 1427-1433 (1998). 
13. D. S. Langley and P. L. Marston, "Glory in optical backscattering from air bubbles," Phys. Rev. Lett. 47, 913-916 (1981).

14. P. L. Marston, "Geometrical and catastrophe optics methods in scattering," in Physical Acoustics, A. D. Pierce and R. N. Thurston, eds. (Academic, Boston, Mass., 1992), Vol. 21, pp. 1-234.

15. R. T. Wang and H. C. van de Hulst, "Rainbows: Mie computations and the Airy approximation," Appl. Opt. 30, 106-117 (1991).

16. H. M. Nussenzveig, Diffraction Effects in Semiclassical Scattering (Cambridge U. Press, Cambridge, 1992).

17. Y. Takano and M. Tanaka, "Phase matrix and cross sections for single scattering by circular cylinders: a comparison of ray optics and wave theory," Appl. Opt. 19, 2781-2793 (1980).
18. F. Kuik, J. F. Dehaan, and J. W. Hovenier, "Single scattering of light by circular cylinders," Appl. Opt. 33, 4906-4918 (1994).

19. M. I. Mishchenko, L. D. Travis, and A. Macke, "Scattering of light by polydisperse, randomly oriented, finite circular cylinders," Appl. Opt. 35, 4927-4940 (1996).

20. M. I. Mishchenko and K. Sassen, "Depolarization of lidar returns by small ice crystals: an application to contrails," Geophys. Res. Lett. 25, 309-312 (1998).

21. Y. G. Liu, W. P. Arnott, and J. Hallett, "Anomalous diffraction theory for arbitrarily oriented finite circular cylinders and comparison with exact T-matrix results," Appl. Opt. 37, 50195030 (1998). 